This is a self-archived version of an original article. This version may differ from the original in pagination and typographic details.

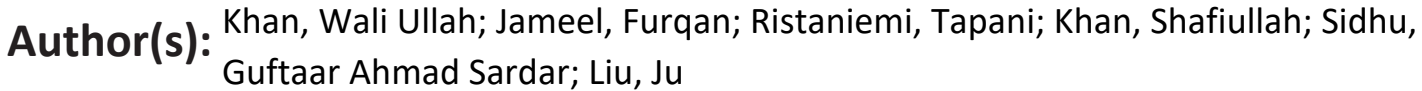

Title: Joint Spectral and Energy Efficiency Optimization for Downlink NOMA Networks

Year: 2020

Version: Accepted version (Final draft)

Copyright: @ 2020 IEEE

Rights: In Copyright

Rights url: http://rightsstatements.org/page/InC/1.0/?language=en

Please cite the original version:

Khan, W. U., Jameel, F., Ristaniemi, T., Khan, S., Sidhu, G. A. S., \& Liu, J. (2020). Joint Spectral and Energy Efficiency Optimization for Downlink NOMA Networks. IEEE Transactions on Cognitive Communications and Networking, 6(2), 645-656.

https://doi.org/10.1109/TCCN.2019.2945802 


\title{
Joint Spectral and Energy Efficiency Optimization for Downlink NOMA Networks
}

\author{
Wali Ullah Khan, Furqan Jameel, Tapani Ristaniemi, Shafiullah Khan, Guftaar Ahmad Sardar Sidhu and Ju Liu
}

\begin{abstract}
Non-orthogonal multiple access (NOMA) holds the promise to be a key enabler of $5 \mathrm{G}$ communication. However, the existing design of NOMA systems must be optimized to achieve maximum rate while using minimum transmit power. To do so, this paper provides a novel technique based on multi-objective optimization to efficiently allocate resources in the multi-user NOMA systems supporting downlink transmission. Specifically, our unique optimization technique jointly improves spectrum and energy efficiency while satisfying the constraints on users quality of services (QoS) requirements, transmit power budget and successive interference cancellation. We first formulate a joint problem for spectrum and energy optimization and then employ dual decomposition technique to obtain an efficient solution. For the sake of comparison, a low complexity single-objective NOMA optimization scheme is also provided as a benchmark scheme. The simulation results show that the proposed joint approach not only performs better than the traditional benchmark NOMA scheme but also significantly outperforms its counterpart orthogonal multiple access (OMA) schemes in terms of both energy and spectral efficiency.
\end{abstract}

Index Terms-Non-orthogonal multiple access, Multi-objective optimization, Spectral efficiency, Energy efficiency, QoS.

\section{INTRODUCTION}

The rapid increase in the demand for high data rates along with energy constrained nature of wireless devices put a great question mark on the capabilities of future communication technologies. It is anticipated that the energy and spectral efficient solutions would play a pivotal role to cope with these challenges. In this regard, non-orthogonal multiple access (NOMA) has emerged as a prominent radio technique to efficiently use the existing resources without compromising the quality of services (QoS) requirements of the users [1], [2]. With the help of superposition coding (SC) techniques at the transmitter and successive interference cancellation (SIC)

This work was supported in part by the National Key R \& D Plan (2017YFC0803403) and the National Natural Science Foundation of China (61371188). (Corresponding author: Ju Liu)

Copyright (c) 2015 IEEE. Personal use of this material is permitted. However, permission to use this material for any other purposes must be obtained from the IEEE by sending a request to pubs-permissions @ieee.org.

Wali Ullah Khan and Ju Liu are with School of Information Science and Engineering, Shandong University, Qingdao, People's Republic of China. (emails: waliullahkhan30@gmail.com, juliu@sdu.edu.cn)

Furqan Jameel is with the Department of Communications and Networking, Aalto University, 02150 Espoo, Finland (emails: furqanjamee101@gmail.com).

Tapani Ristaniemi is with the Faculty of Information Technology, University of Jyväskylä, FI-40014 Jyväskylä, Finland. (emails: tapani.ristaniemi@jyu.fi)

Shafiullah Khan is with Department of Electronics, Islamia College University, Peshawar, Pakistan. (email: shafielectronics@icp.edu.pk)

Guftaar Ahmad Sardar Sidhu is with Department of Electrical Engineering, COMSATS University, Islamabad, Pakistan. (email: guftaarahmad@comsats.edu.pk) methods at the receiver, NOMA schemes can accommodate multiple users by utilizing the same spectrum and time resources [3], [4].

\section{A. Related Work}

Due to the remarkable ability of NOMA schemes, researchers have recently studied energy efficiency in NOMA systems. In this regard, Shi et al. in [5] presented an energy efficient resource management scheme for hybrid NOMA wireless networks, where two different power control and user clustering techniques were compared. The work in [6] investigated a beamforming technique for multiple-input-singleoutput (MISO) NOMA system to increase energy efficiency. The authors first transformed the optimization problem into a convex problem and then exploited two algorithms based Dinkelbach's and sequential convex programming approaches. A resource optimization problem was proposed to improve the energy efficiency of NOMA heterogeneous networks under the consideration of both perfect and imperfect channel state information (CSI) in [7]. They first performed user scheduling under equal power consideration and then employed a fractional power control policy to solve the convex optimization problem. Liu et al. in [8] investigated an energy maximization scheme for cooperative NOMA system. The original nonconvex problem for joint power optimization at source and relay was decomposed into the two-layered optimization problem to obtain the optimal solutions. A resource management problem to increase energy efficiency was studied in NOMA networks supporting device-to-device (D2D) transmission [9]. They adopted a low complex Karush-Kuhn-Tucker (KKT) based iterative algorithm to obtain the efficient solution. The authors of [10] proposed an energy efficient resource management problem for multi-user NOMA systems to improve the overall system energy efficiency. They provided a sub-optimal technique for sub-channel and power allocation. In a similar manner, a power optimization technique was investigated to enhance the energy efficiency in multi-user NOMA systems [11]. To solve the non-convex problem, they derived a closedform expression followed by KKT conditions. A resource optimization scheme to increase the energy efficiency of multiuser NOMA network was investigated in [12]. The joint problem of power and user allocation was formulated as nonconvex where they first transformed it to a nonprobabilistic problem and then adopt an iterative approach to obtain an efficient solution. In [13], Zhang et al. solved the problem of sub-channel and power allocation in order to improve the energy efficiency in NOMA networks. The sub-optimal 
algorithms were employed based on the two-sided matching technique for optimal sub-channel assignment and Lagrangian optimization for efficient power allocation. Besides this, the problem of efficient power allocation was also considered by Ni et al. in [14]. They performed user clustering and optimal power management to reduce the total power consumption of multi-cell NOMA networks. Of late, the problems of joint sub-channel and power management were investigated in [15][17], wherein, the authors maximized the energy efficiency for downlink heterogeneous networks employing NOMA scheme.

Besides energy efficiency, researchers have also focused on the spectral efficiency aspect of NOMA networks. For instance, Khan et al. in [3] proposed an efficient power allocation problem for spectral efficiency in multi-cell multi-user NOMA systems. They employed a tractable sub-optimal technique to obtain an efficient solution. A resource optimization scheme was provided to enhance the sum rate of NOMA systems supporting multi-carrier communications [18]. The problems of user association with the base station (BS), sub-channel assignment and power management were formulated as mixed integer programming. Here, they first performed the user association and sub-channel assignment by three-dimensional (3D) matching approach and then adopted efficient power management through brand and bound (BB) approach. Sun et al. in the multi-cell scenario provided a joint solution for optimal beamforming and power allocation in a network supporting NOMA in power multiplexing [19]. They divided the number of users in two different groups based on minimum rate requirements. The non-convex optimization problem of sum rate maximization was solved by sub-optimal successive convex approximation algorithm. Chen et al. in [20] proposed a power allocation and spectrum sharing problem for two slot secondary NOMA relay networks to increase the sum rate of cellular users. The data symbols for primary and secondary receivers were first transmitted from NOMA relay and then data symbol for the primary receiver was retransmitted from secondary receiver to improve its QoS. Besides this, a problem was considered in downlink MISO NOMA networks to enhance the system sum rate [21]. The non-linear problem was first approximated with the minorization-maximization algorithm then they solved the second-order cone program with polynomial computational complexity in each step. In another study, Zhang et al. presented a resource management problem for amplify-and-forward (AF) relaying NOMA network [22]. The objective was to increase the system sum rate through sub-channel and power optimization at source/ destination pairs. To tackle with the non-linear problem, they employed sub-optimal solutions based on matching theory and waterfilling algorithms. In [23], Fu et al. investigated a resource management scheme in multi-carrier network to increase the sum network capacity. They proposed a three-step water-filling algorithm for sub-carrier and power optimization to different users in the network. A joint problem for beamforming and power management was presented by Xue et al. in multiuser NOMA network to increase the user achievable data rate [24]. The optimization problem was first approximated as a convex problem and then solved by semidefinite programming based one-dimensional search algorithm for the case of two
Table I

THE LIST OF DIFFERENT SYMBOLS AND THEIR DEFINITIONS

\begin{tabular}{l|l}
\hline Symbol & Definition \\
\hline$M$ & Number of users. \\
$U_{m}$ & Index of $m$ th user. \\
$y_{m}$ & Received signal of $U_{m}$. \\
$h_{m}$ & Channel gain of $U_{m}$. \\
$p_{m}$ & Transmit power of $U_{m}$. \\
$s_{m}$ & Data symbol of $U_{m}$. \\
$\omega_{m}$ & AWGN of $U_{m}$. \\
$\sigma^{2}$ & Variance of AWGN. \\
$R_{m}$ & Achievable rate of $U_{m}$. \\
$\tau_{m}$ & SINR of $U_{m}$. \\
$\theta$ & Minimum power gap for successful SIC. \\
$\bar{R}_{m i n}$ & Minimum required rate for QoS. \\
$t$ & Iteration index. \\
$\lambda_{m}$ & Lagrange multiplier . \\
$\mu_{m}$ & Lagrange multipliers. \\
$\eta_{m}$ & Lagrange multiplier . \\
$\mathcal{L}$ & Lagrangian function. \\
$\Lambda$ & Dual problem. \\
$\delta$ & Non-negative step size. \\
\hline
\end{tabular}

Table II

THE LIST OF DIFFERENT ABBREVIATIONS AND THEIR DEFINITIONS

\begin{tabular}{l|l}
\hline Acronym & Definition \\
\hline SIC & Successive interference cancellation. \\
5G & Fifth generation. \\
D2D & Device-to-Device. \\
OMA & Orthogonal multiple access. \\
3D & Three-dimensional. \\
BB & Brand and bound. \\
NOMA & Non-orthogonal multiple access. \\
MISO & Multiple-input single-output. \\
SC & Superposition coding. \\
AF & Amplify-and-forward. \\
CSI & Channel state information. \\
BS & Base station. \\
KKT & Karush-Kuhn-Tucker. \\
LCM & Least common multiple. \\
PC & Circuit power. \\
QoS & Quality of services. \\
AWGN & Additive white Gaussian noise. \\
\hline
\end{tabular}

users. Reference [25] jointly optimized sub-channel and power allocation for multi-user NOMA networks. The aim of this work was to increase the sum rate of the system. The joint solution was obtained through a matching game and interior point methods. Similarly, Liang et al. considered a resource allocation problem to enhance the user average data rate in cooperative NOMA network [26]. To tackle the NP-hard problem, they first decoupled the joint resource allocation problem into two separate sub-problems and then adopted two sub-optimal schemes based on matching game approach for the user sub-channel association and dual method for efficient power allocation. In addition, the problems of resource management to increase the spectral efficiency in NOMA heterogeneous networks were also investigated in [27]-[30].

To date, all related research works in NOMA networks have focused on single-objective optimization either optimization of spectral efficiency or energy efficiency. However, in several situations, decision makers find themselves need to optimize multiple functions with different objectives simultaneously which raises the requirement of multi-objective optimization 
[31]. Multi-objective optimization is an optimization technique which involve more than one objectives that are to be maximized or minimized simultaneously [32]. In this work, we employ multi-objective optimization technique where optimal decisions need to be taken in the presence of tradeoff among conflicting spectrum and energy efficient objectives [33]. In general, it is difficult to obtain a single solution which simultaneously optimizes multi-objective functions. More specifically, no solution exists which enhance one of the objective without degrading other objective functions, the condition is called Pareto optimality [34]. However, there exists a set of non-dominated, weak Pareto optimal solutions and it is the user's responsibility to choose its preferred optimal solution. Different techniques for solving multi-objective optimization problems exist and are categorized according to the level of preferences of the competing objective functions [34]. In this paper, we exploit the weighted-sum method, where the objective of spectral and energy efficiency can be linearly combined as a single-objective function. This method uses a set of positive weighting coefficients which reflect the users preferences according to the target performance, the application, and the surrounding environment.

\section{B. Motivation and Contributions}

The implementation of NOMA in power multiplexing depends on the guaranteed SIC operation. However, to successfully complete the SIC at users, a sufficient gap between users transmit powers is required and should be guaranteed by the proposed solutions. Unfortunately, the recent studies [2]-[30] lack this practical consideration. In fact, these studies do not guarantee that such a gap exists among users' transmit powers in their proposed frameworks. Additionally, the focus of many of NOMA studies is on single-objective optimization that most often include maximization of energy efficiency or spectral efficiency under conventional constraints. Adopting a different approach from existing studies, our work employs multiobjective optimization to obtain the most preferred optimal solution among multiple and contradicting objective functions. Furthermore, we have considered the constraints on minimum user rate, total BS power and the minimum gap between users powers to accomplish the SIC process. The salient aspects of our multi-objective optimization method are five-fold and outlined as follows.

1) By exploiting power domain NOMA principle, we consider a joint optimization of spectral and energy efficiency in downlink NOMA networks. The objective is to simultaneously increase the user sum rate and to reduce the transmit power of each user at BS while satisfying each user QoS requirement, transmit power budget and successful SIC constraints.

2) To achieve the joint performance improvements for rate and transmit power, we provide a technique based on multi-objective optimization. To date, all related works in NOMA networks have considered single-objective optimization problems. Thus, to the best of our knowledge, this is the first NOMA work which considers multiobjective optimization for the said metrics.

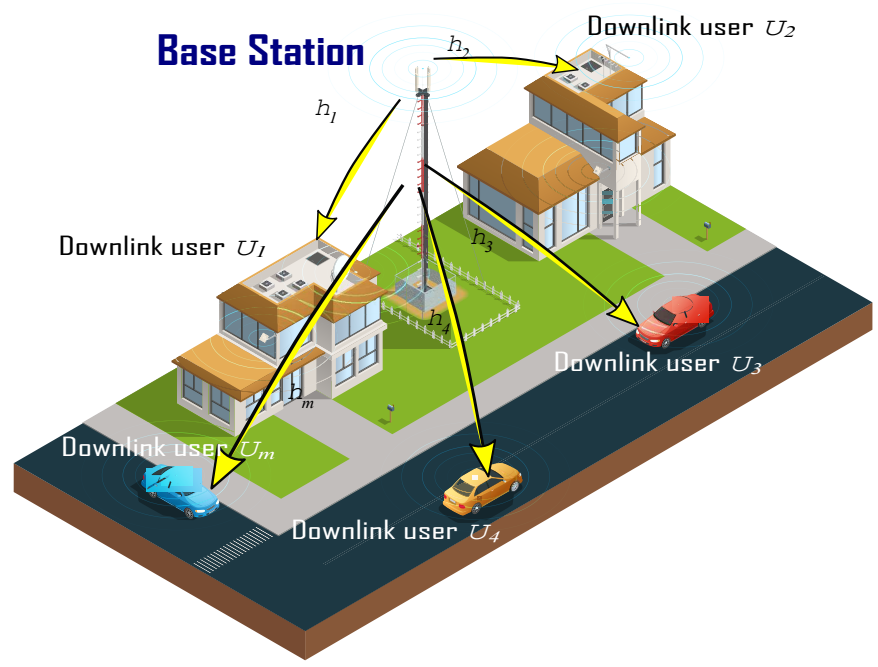

Figure 1. System model of NOMA network supporting downlink transmission

3) Unlike the solutions discussed in [2]-[30] which do not consider the minimum gap among users powers to perform successful SIC technique, we guarantee the SIC process by considering the constraint of minimum gap among users transmit powers to our joint framework.

4) To derive the optimal solution for our multi-objective convex problem, we adopt a dual theory which satisfy KKT conditions, where Lagrange multipliers can be obtained and iteratively updated using the sub-gradient method. For a fair comparison, we also consider a low complexity single-objective NOMA optimization scheme as a benchmark.

5) We perform Monte-Carlo simulation where we obtain the average from $10^{5}$ realizations. The results depict that the proposed joint approach not only performs better than the traditional benchmark single-objective NOMA optimization scheme but also significantly outperforms its counterpart optimal OMA scheme. In addition, we find that our NOMA scheme gives good performance for high $\mathrm{QoS}$ requirements and take only a few iterations in convergence.

The rest of the paper is organized as follows: The system description and different formulation steps are provided in Section II. In Section III, we discuss the optimal solution to the problem for downlink NOMA while Section IV presents the numerical results and the relevant discussion. Finally, Section $\mathrm{V}$ provides concluding remarks and future work. The definition of different acronyms and abbreviations used in our work are respectively defined in Table I and II.

\section{System Model and Joint Problem Formulation}

This section provides system model and, subsequently, discusses the problem formulation steps.

Consider a downlink NOMA network as illustrated in Fig. 1 , having a BS with $M$ different users, where $\mathcal{U}=\left\{U_{m} \mid m=\right.$ $1,2,3, \ldots M\}$. Utilizing the power multiplexing of NOMA, 


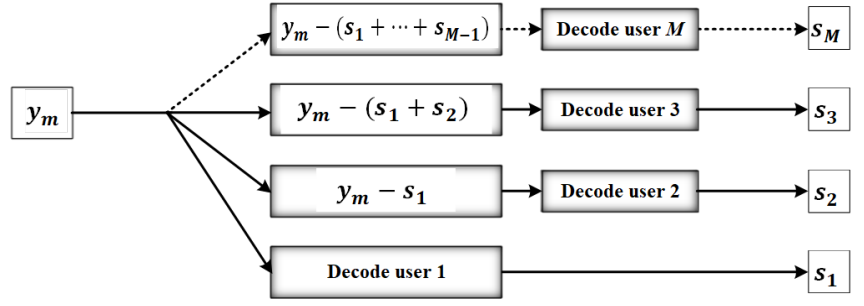

Figure 2. The SIC process in $M$ users downlink NOMA transmission.

all users are accommodated over the same frequency band ${ }^{1}$, whereby, the BS and users follow a single antenna configuration. Since the users are connected to the BS, we consider that BS has the knowledge of CSI of all its serving users [35]. In addition, we consider the Rayleigh fading channel between BS and all its serving users. To perform successful SIC process at downlink transmission, we sort all the users based on their channel gains such that $\left|h_{1}\right| \leq\left|h_{2}\right| \leq \ldots \leq\left|h_{M}\right|$. According to this order, $U_{1}$ decodes its signal first followed by $U_{2}$ and so on. Thus, $U_{1}$ cannot apply SIC and decodes its signal with the interference of all other users, while $U_{M}$ decode its signal after eliminating interference from all other users by applying the SIC technique. Fig. (2) illustrates the SIC process of downlink NOMA transmission. After the SIC process, the received signal of $U_{m}$ is given by

$$
y_{m}=h_{m} \sqrt{p_{m}} s_{m}+h_{m} \sum_{n=m+1}^{M} \sqrt{p_{n}} s_{n}+\omega_{m},
$$

where $h_{m}$ is the channel gain of $U_{m}, p_{m}$ represents the $U_{m}$ transmit power, and $s_{m}$ is the data symbol of $U_{m}$. The second term in (1) denotes the interference of other users using the same spectrum resource after the SIC process. The noise term $\omega_{m} \sim \mathcal{C N}\left(0, \sigma^{2}\right)$ is the zero mean additive white Gaussian noise (AWGN) with variance $\sigma^{2}$. For the received signal of $U_{m}$ derived in (1), the data rate is

$$
R_{m}=\log _{2}\left(1+\tau_{m}\right),
$$

where $\tau_{m}$ in (2) is the signal-to-interference and noise ratio (SINR) of $U_{m}$ and can be given by

$$
\tau_{m}=\frac{p_{m}\left|h_{m}\right|^{2}}{\sum_{n=m+1}^{M} p_{n}\left|h_{m}\right|^{2}+\sigma^{2}} .
$$

To make the SIC process successful in the downlink transmission, the transmit power of all the users must satisfy the minimum required gap as [36]

$$
\left(p_{m}-\sum_{n=m+1}^{M} p_{n}\right)\left|h_{m+1}\right|^{2} \geq \theta,
$$

where $\theta$ is the minimum power gap among different users to apply SIC technique successfully. Following the condition in (4), the transmit powers of $M$ users should satisfy as $p_{1}>p_{2}>\ldots>p_{M}$. Note that the SIC constraints for $M$

\footnotetext{
${ }^{1}$ In this work, we consider the same spectrum resource for all users of the NOMA network, the problem of multiple spectrum resources using multiobjective optimization is set aside for our future work.
}

users NOMA, where all users are communicated over the same spectrum resource will be reduced exactly to $(M-1)$. It is worth highlighting that for $M$ users NOMA systems, where all users are multiplexed on the same spectrum resource with any specific SIC ordering, the strongest user generally applies SIC and removes all other users' signals before decoding its own signal. In the end, the weakest user decodes the signal without using SIC technique by treating all other users' signals as an interference. This is the reason why the SIC constraints will be on $M-1$ under NOMA communications conditions.

We intend to reduce the user power consumption and improve the sum rate while satisfying the individual QoS requirement for all users. The maximum sum of user rate and the minimum total transmit power of BS in the downlink transmission leads to multiple objectives. It can be achieved by solving the multi-objective optimization. This multi-objective optimization problem can be written as (P1)

$$
\begin{array}{ll}
\text { (P1) } & \max _{p_{m}} \sum_{m=1}^{M} R_{m}, \\
& \min _{p_{m}} \sum_{m=1}^{M} p_{m}, \\
\text { s.t. } \quad & R_{m} \geq R_{\min }, \forall m, \\
& \sum_{m=1}^{M} p_{m} \leq P_{B S}, \\
& \left(p_{m}-\sum_{n=m+1}^{M} p_{n}\right)\left|h_{m+1}\right|^{2} \geq \theta, \\
& \forall m \in\{1,2, \ldots, M-1\}, \\
& p_{m} \geq 0, \forall m .
\end{array}
$$

where (5a) and (5b) are the objective functions for sum rate maximization and total transmit power minimization, respectively. Constraint in $(5 \mathrm{c})$ ensures per user minimum data rate where $R_{\min }$ is the threshold for $U_{m}$ to achieve the minimum data rate. Constraint in (5d) limits the total power of BS where $P_{B S}$ is the BS total power budget. Additionally, the constraint in $(5 \mathrm{e})$ guarantees successful completion of the SIC process in the downlink transmission while constraint in (5f) shows non-negative transmit power of $U_{m}$.

\section{Proposed Optimal Solutions}

This section will provide the optimal solution of $M$ users downlink NOMA transmission in the joint metrics of sum spectral efficiency and total network energy efficiency. These metrics can be achieved by joint optimization of optimal power allocation at the source using multi-objective optimization technique. Later, we also discuss a single-objective NOMA optimization as benchmark scheme.

\section{A. Proposed Joint Optimization Scheme}

To calculate the joint optimization of optimal power management for downlink transmission, we now apply the weighted-sum method to deal with the multi-objective optimization problem explained in (P1). This is one of the 
most successful tool for solving such kind of optimization problems [37]. According to this method, multiple objectives can be linearly combined as a single-objective optimization by employing a weighting coefficient which indicates the tradeoff between multi-objectives [34], [38], [39]. By applying this technique, the formulated problem (P1) can be modified as (P1.1):

$$
\begin{aligned}
& \max _{p_{m}} \beta\left(\sum_{m=1}^{M} R_{m}\right)-(1-\beta)\left(\sum_{m=1}^{M} p_{m}\right), \\
& \text { s.t. } \quad(5 c),(5 d) \text { and }(5 f) .
\end{aligned}
$$

where $\beta$ is the weighting coefficient such as $0 \leq \beta \leq 1$ which indicates the tradeoff between two objectives, i.e., higher values of $\beta$ favor maximizing the system spectral efficiency, while lower values of $\beta$ favor the system energy efficiency. In addition, we suppose that the users select the proper values of $\beta$ based on the mode of operations. For instance, when the data rate, and, hence, spectral efficiency is important, then the users choose the higher values of $\beta$. However, if the transmit power, and energy efficiency is more crucial, then lower values of $\beta$ are selected.

Proposition 1. For $M$ users downlink NOMA network with any specific SIC ordering, the problem in (P1.1) is convex.

Proof: Please check Appendix A for the solution.

As shown in Appendix A, the multi-objective problem (P1.1) is concave-convex. This observation motivates us to use dual decomposition methods to get the optimal solution. To use employ this method, we first derive the Lagrangian function such as

$$
\begin{aligned}
& \mathcal{L}\left(p_{m}, \lambda_{m}, \mu_{m}, \eta_{m}\right)=\beta\left(\sum_{m=1}^{M} R_{m}\right)-(1-\beta)\left(\sum_{m=1}^{M} p_{m}\right) \\
& +\sum_{m=1}^{M} \lambda_{m}^{d}\left(R_{m}-R_{\min }\right)+\mu_{m}^{d}\left(P_{B S}-\sum_{m=1}^{M} p_{m}\right) \\
& +\sum_{m=1}^{M-1} \eta_{m}\left(\left(p_{m}-\sum_{n=m+1}^{M} p_{n}\right)\left|h_{m+1}\right|^{2}-\theta\right),
\end{aligned}
$$

where $\lambda_{m}, \mu_{m}$ and $\eta_{m}$ are the positive Lagrangian multipliers. Using this expression, the dual problem of optimization problem (P1.1) can be derived as

$$
\begin{aligned}
& \min _{\lambda_{m}, \mu_{m}, \eta_{m}} \Lambda\left(\lambda_{m}, \mu_{m}, \eta_{m}\right), \\
& \text { s.t. } \quad \lambda_{m} \geq 0, \mu_{m} \geq 0, \eta_{m} \geq 0,
\end{aligned}
$$

where

$$
\begin{aligned}
& \Lambda\left(\lambda_{m}, \mu_{m}^{d}, \eta_{m}\right)=\max _{p_{m}} \beta\left(\sum_{m=1}^{M} R_{m}\right)-(1-\beta)\left(\sum_{m=1}^{M} p_{m}\right) \\
& +\sum_{m=1}^{M} \lambda_{m}\left(R_{m}-R_{\min }\right)+\mu_{m}\left(P_{B S}-\sum_{m=1}^{M} p_{m}\right) \\
& +\sum_{m=1}^{M-1} \eta_{m}\left(\left(p_{m}-\sum_{n=m+1}^{M} p_{n}\right)\left|h_{m+1}\right|^{2}-\theta\right)
\end{aligned}
$$

Note that the difference between dual and primal solution has been proved to be zero (see [13] and references therein).
Now, by applying KKT conditions, the closed-form expression for $p_{m}$ has been derived in Appendix B as

$$
p_{m}^{*}=\left[\frac{\left(\beta_{m}+\phi_{m}\right)\left|h_{m}\right|^{2}-\left(\phi_{m}\right)\left|h_{m}\right|^{2} p_{n}-\sigma^{2}\left(\phi_{m}\right)}{\left|h_{m}\right|^{2}\left(\phi_{m}\right)}\right]^{+},
$$

where $(\alpha)^{+}=\max (0, \alpha)$, and

$$
\begin{aligned}
\phi_{m} & =\sum_{n=1}^{m-1} \eta_{n}\left|h_{n}\right|^{2}+\mu_{m}+1-\beta-\left(\beta+\lambda_{n}\right) \\
& \times\left(\sum_{l=1}^{n-1} \frac{-\left|h_{l}\right|^{2}}{\sum_{i=l+1}^{l-1} p_{i}\left|h_{l}\right|^{2}+\sigma^{2}}\right) .
\end{aligned}
$$

With optimal value of $p_{m}$, our dual problem becomes

$$
\begin{aligned}
& \min _{\lambda_{m}, \mu_{m}, \eta_{m}} \beta\left(\sum_{m=1}^{M} R_{m}^{*}\right)-(1-\beta)\left(\sum_{m=1}^{M} p_{m}^{*}\right) \\
& +\sum_{m=1}^{M} \lambda_{m}\left(R_{m}^{*}-R_{\min }\right)+\mu_{m}\left(P_{B S}-\sum_{m=1}^{M} p_{m}^{*}\right) \\
& +\sum_{m=1}^{M-1} \eta_{m}\left(\left(p_{m}^{*}-\sum_{n=m+1}^{M} p_{n}\right)\left|h_{m+1}\right|^{2}-\theta\right), \\
& \text { s.t. } \quad \lambda_{m} \geq 0, \mu_{m} \geq 0, \eta_{m} \geq 0 .
\end{aligned}
$$

Now the dual variables in (12) can be obtained and iteratively updated using sub-gradient method in [40], [41] as

$$
\begin{aligned}
\lambda_{m}(t+1) & =\left[\lambda_{m}(t)+\delta(t) \times\left(R_{\min }-R_{m}\right)\right]^{+}, \forall m, \\
\mu_{m}(t+1) & =\left[\mu_{m}(t)+\delta(t) \times\left(\sum_{m=1}^{M} p_{m}-P_{B S}\right)\right]^{+}, \\
\eta_{m}(t+1) & =\left[\eta_{m}(t)+\delta(t) \times\left(\theta-\left(p_{m}-\sum_{n=m+1}^{M} p_{n}\right)\right.\right. \\
& \left.\left.\times\left|h_{m+1}\right|^{2}\right)\right]^{+}, \forall m \in\{1,2, \ldots, M-1\},
\end{aligned}
$$

where $t$ and $\delta \geq 0$ represent the iteration index and step size, respectively. In each number of $t, \lambda_{m}, \mu_{m}$ and $\eta_{m}$ are updated using $p_{m}^{*}$ obtained from (10). In the subsequent iterations, the optimal values of $\lambda_{m}, \mu_{m}$ and $\eta_{m}$ are used to calculate $p_{m}^{*}$. The iterative process continues until convergence.

Now, we discuss the complexity of our multi-objective optimization technique. This is based on the number of iterations which our scheme needs for the convergence. The number of iterations required for convergence will be increased if high number of users are sharing the same spectrum resource at the same time. Since we consider $M$ users in our proposed NOMA network, the complexity for dealing with problem (P1.1) using multi-objective optimization technique in each iteration is $\mathcal{O}\left(M^{2}\right)$. We assume that the total iteration are $T$, then the total computational complexity of our proposed joint framework becomes $\mathcal{O}\left(T M^{2}\right)$. 


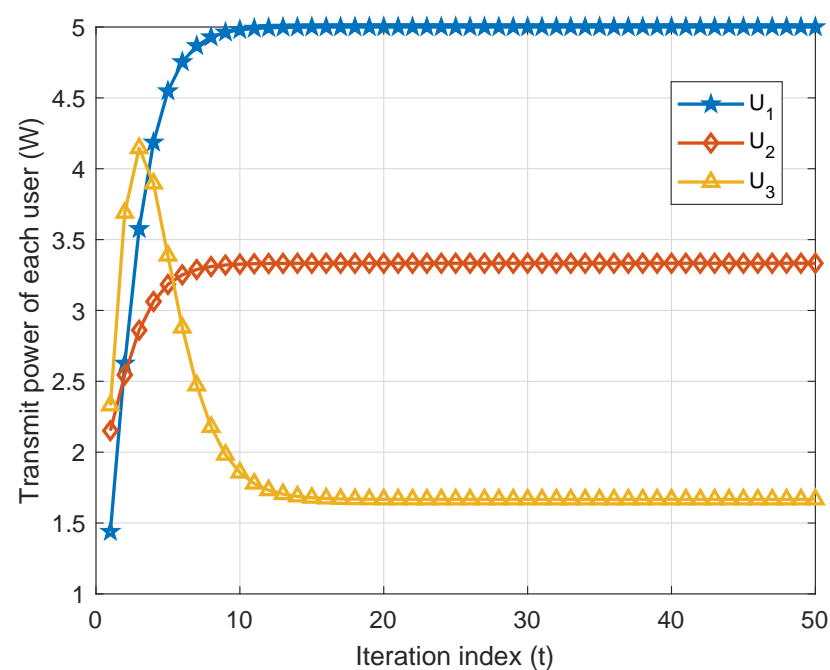

Figure 3. Number of iterations versus the power allocation of each user according to SIC constraint for $M=3, \mathrm{PC}=1, P_{B S}=10 \mathrm{~W}, \beta=1$.

\section{B. Benchmark Single-objective NOMA Scheme}

Section III-A discusses a joint spectral and energy optimization for $M$ users NOMA network. In this subsection, to reduce the computational complexity, we present a single-objective optimization scheme as a benchmark where power allocation is optimized for sum rate maximization only. The power optimization problem can be reduced to sum rate optimization such as (Ps):

$$
\begin{array}{ll}
\text { (Ps) } & \max _{p_{m}} \sum_{m=1}^{M} R_{m}, \\
\text { s.t. } & R_{m} \geq \bar{R}_{\min }, \forall m, \\
& \sum_{m=1}^{M} p_{m} \leq P_{\mathrm{BS}}, \\
& \left(p_{m}-\sum_{n=m+1}^{M} p_{n}\right)\left|h_{m+1}\right|^{2} \geq \theta, \\
& \forall m \in\{1,2, \ldots, M-1\}, \\
& p_{m} \geq 0, \forall m .
\end{array}
$$

The problem (Ps) is convex [42], and we employ the same technique with similar steps as discussed for multi-objective optimization scheme in Section III- $A$, however all the steps involve in the convexity proof and solutions of the problem are missing for the sake of simplicity.

\section{Results, COMPARISON AND Discussion}

This section provides the simulation results of our proposed multi-objective optimization technique, as discussed in Section III- $A$ as well as the benchmark single-objective optimization NOMA scheme, as discussed in Section III- $A$. In addition, we also provide the results of conventional OMA technique based on multi-objective optimization. We performed linklevel simulations in MATLAB and took $10^{5}$ realization of channel gains to generate each data point on the forthcoming

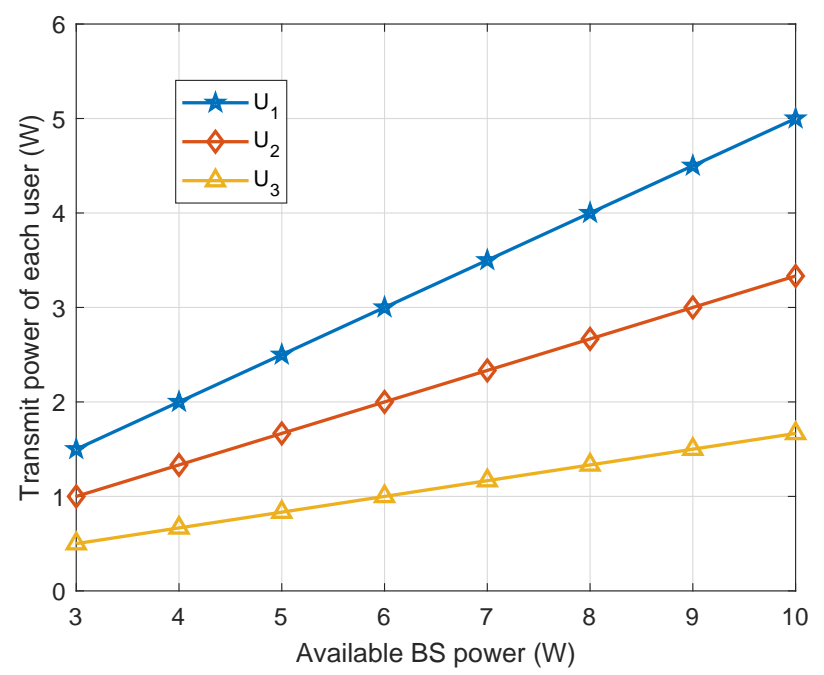

Figure 4. Available BS transmit power versus the power allocation of each user according to SIC constraint for $M=3, \mathrm{PC}=1, \beta=1$.

Table III

VALUES OF DIFFERENT PARAMETERS USED FOR SIMULATION.

\begin{tabular}{|l||l|}
\hline Parameter & Definition \\
\hline \hline Number of active users & $M=3$ \\
Maximum power (W) & 10 \\
Channel type & Raleigh fading \\
$\beta$ & $0.6-1$ \\
PC (W) & 1 \\
$\sigma^{2}$ & 1 \\
$\theta$ & 0.5 \\
Channel realization & $10^{5}$ \\
\hline
\end{tabular}

plots. Unless mentioned otherwise, the values of the simulation parameters used to generate the plots are listed in Table III.

\section{A. Impact of SIC Constraint on Power Allocation}

For successful implementation of NOMA exploiting power multiplexing, the successful operations of SIC is mandatory and must be guaranteed. For this, it is necessary that received signal powers of different users have the minimum gap. These power gap should be assign according to the users channel conditions. Moreover, by using this gap, a user employing SIC can decide which signal needs to decode and which one to subtract.

In Fig. 3, we have illustrated the transmit power of different users as a function of the number of iterations. Since we assume to order the users channel power in ascending order, i.e., $\left|h_{1}\right|^{2} \leq\left|h_{2}\right|^{2} \leq\left|h_{3}\right|^{2}$, which means that $U_{3}$ has strong channel gain, followed by $U_{2}$ and at the end $U_{1}$ has the weakest channel gain. We can see from the plot, the condition of NOMA protocol, i.e., $p_{1}>p_{2}>p_{2}$, means $U_{3}$ has assigned the least transmit power, followed by $U_{2}$ and at the end $U_{1}$ has allocated the largest transmit power. Furthermore, it can be seen that for user 1 and 2, the transmit power first increases and then remains unchanged as the number of iterations exceeds 15. However, for user 3, the transmit power first increases up to $4.3 \mathrm{~W}$ and then decreases to 1.7 W. Afterward, the power for the third user remains constant as 


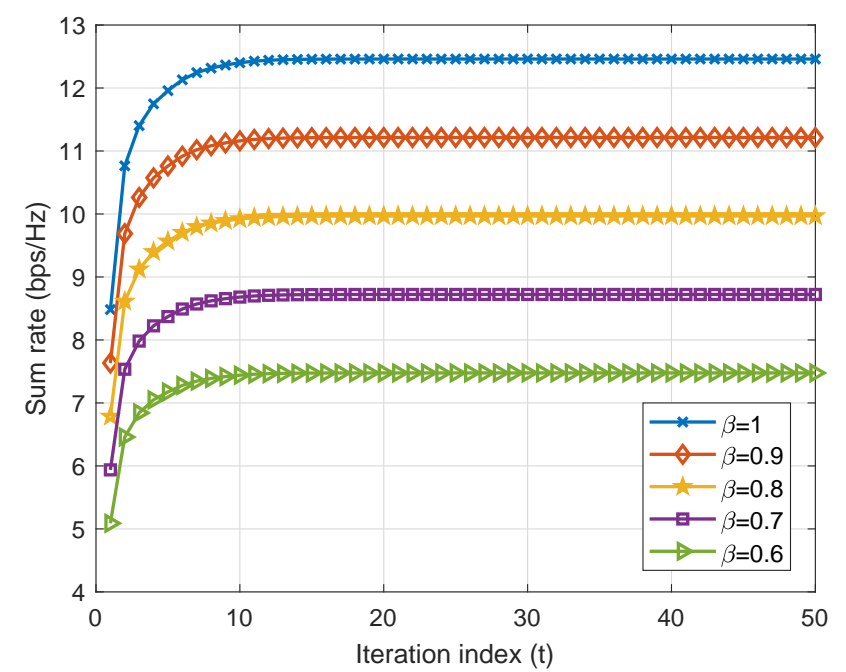

Figure 5. The sum rate of the NOMA system with different values of $\beta$ versus number of iterations when $M=3, \mathrm{PC}=1, P_{B S}=10$.

the number of iterations exceeds from 15 . These variations and trends in the transmit powers of different users are due to the sorted channel gains and subsequently the allocation of power according to the SIC constraint, as discussed above. In addition to this, note that the number of iterations for the proposed methodology is within a reasonable range, even for 3 users in the networks. This clearly highlights the lightweight and less complex nature of our proposed NOMA multi-objective optimization scheme.

Fig. 4 further emphasizes the operations of SIC constraint to control the transmit power of different users according to the NOMA protocol. Here, we plot the transmit power of different users against the increasing values of BS available power. It can be seen that the transmit power of the users increases when the available BS power increases. From the figure, one can observe that due to SIC condition, the users keep a minimum gap between their transmit powers. More specifically, for fixed value of BS transmit power, for example, when $P T=3 \mathrm{~W}$, the transmit power of $U_{3}$ is $0.5 \mathrm{~W}$, followed by $U_{2}$ with $1 \mathrm{~W}$ and the power of $U_{1}$ is $1.5 \mathrm{~W}$. Moreover, the gap between users transmit powers increasing with increase in the BS available transmit power. This is because the minimum gap also increases with higher values of BS available power.

\section{B. Spectral Efficiency Aspects}

In Fig. 5, we have illustrated the spectral efficiency of $M$ users in downlink NOMA network versus the number of iterations. From the figure, it can be seen that for different values of $\beta$, the sum rate first increases and then remains unchanged as the number of iterations passes 15 . Note that the total iterations required for our proposed multi-objective NOMA methodology is within a reasonable range. This signifies the lightweight nature and low complexity of our proposed optimization method.

Fig. 6 depicts the sum rate of the $M$ users in a downlink network against increasing values of the available BS

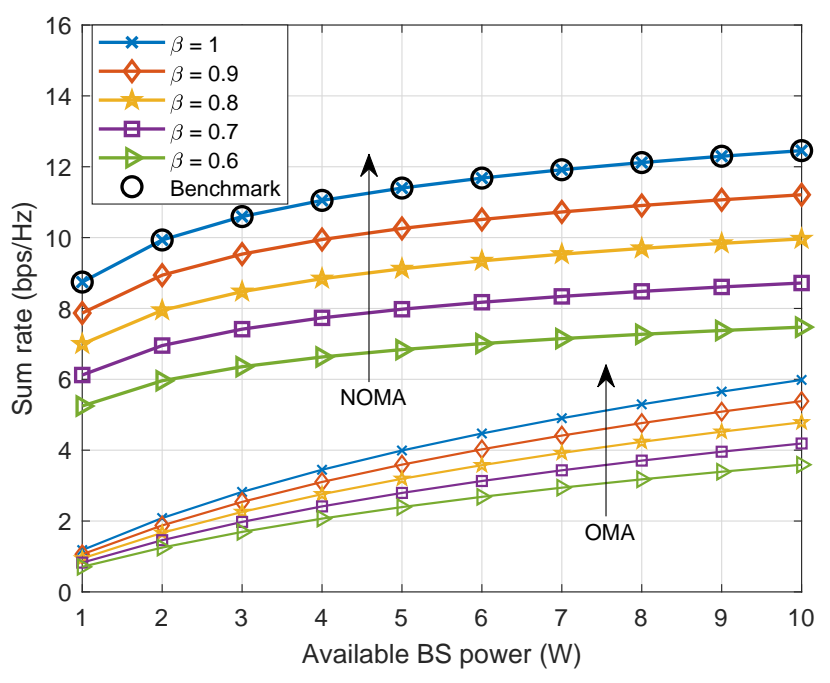

Figure 6. The sum rate of the NOMA system versus the available BS power for $M=3, \mathrm{PC}=1$.

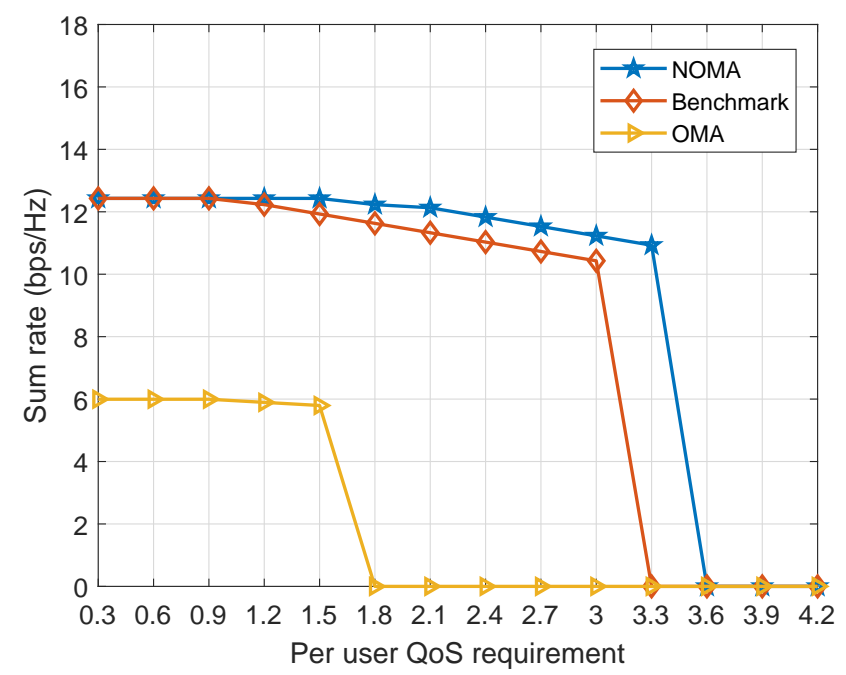

Figure 7. The sum rate of the network versus per user QoS requirement for $P_{B S}=10, \beta=1, M=3, \mathrm{PC}=1$.

transmit power. We can observe the increase in system sum rate for increasing values of BS available transmit power. As is evident from (P1.1), an increase in $\beta$ increases the sum rate of the system for both NOMA and OMA schemes. For fair comparison, we have also demonstrated the results for NOMA technique when single-objective optimization is applied (represented as "Benchmark"). The benchmark scheme is aimed to improve the sum rate of the downlink system due to which it underperforms for the case of energy efficiency. We can see that the curve of the benchmark NOMA scheme closely follows the curve of $\beta=1$ which illustrates the dependency of sum rate on different values of $\beta$. In contrast, we observe that for a fixed transmit power $(10 \mathrm{~W})$, when $\beta$ increases from 0.6 to 0.9 , the sum rate for $M$ users downlink NOMA increases from 7.5 to $12.5 \mathrm{bps} / \mathrm{Hz}$. In contrast, for the similar values of $\mathrm{BS}$ available power and $\beta$, the data rate for 


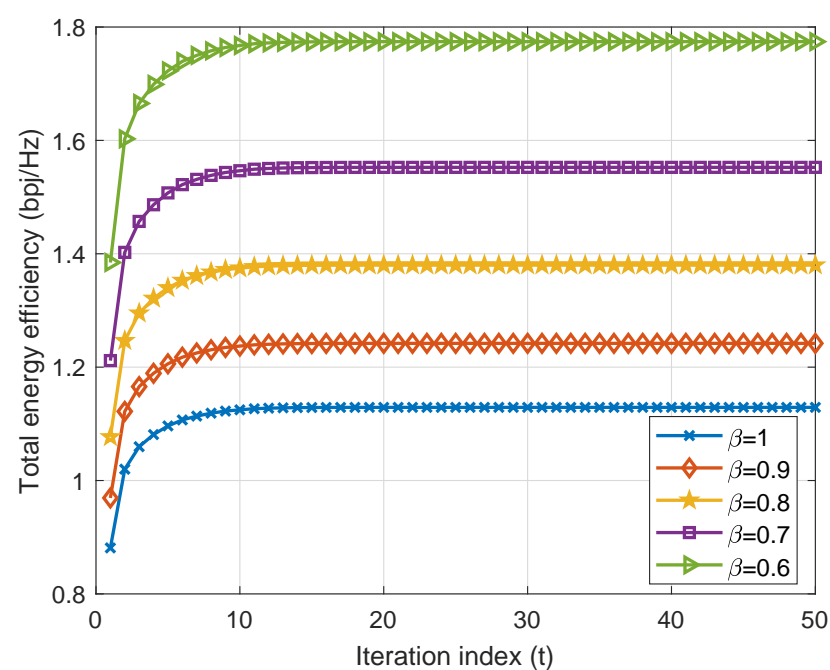

Figure 8. Total energy efficiency of the network with different values of $\beta$ versus number of iterations when $M=3, \mathrm{PC}=1, P_{B S}=10$.

OMA minutely increases from 3.5 to $6 \mathrm{bps} / \mathrm{Hz}$. This shows that our proposed method not only performs better for multiuser NOMA but also improves the sum rate in comparison to OMA.

Fig. 7 depicts the sum rate of the NOMA network against increasing values of per-user QoS requirement. As demonstrated before, one can observe here that our multi-objective optimization scheme outperforms the single-objective optimization and conventional OMA schemes. By keeping the QoS fixed at $\bar{R}_{\min }=1.5 \mathrm{bps} / \mathrm{Hz}$ and keeping $P_{\max }=10$, the sum rate of the joint NOMA scheme is $12.4 \mathrm{bps} / \mathrm{Hz}$ while for the same values, the sum rate of the benchmark is $11.9 \mathrm{bps} / \mathrm{Hz}$ and the OMA is $5.8 \mathrm{bps} / \mathrm{Hz}$, respectively. Further, the sum rate of the conventional OMA scheme drops to zero when $\bar{R}_{\text {min }}$ approaches the value of $1.8 \mathrm{bps} / \mathrm{Hz}$. This is because the available transmit power is not large enough to satisfy the high QoS demand of OMA users. In addition, the rate gap between joint NOMA and benchmark NOMA increase for high values of QoS requirements. This change illustrates the positive impact of using multi-objective optimization as the proposed scheme continues to perform well even at higher QoS requirements.

\section{Energy Efficiency Aspects}

In Fig. 8, we have plotted the number of iteration versus the total energy efficiency of $M$ users in downlink NOMA network. We can see from the plot that for different values of $\beta$, the curves of total energy efficiency against different values of $\beta$ first increases and then remains unchanged as the number of iteration exceeds from 15. In addition, the number of iterations requires for the proposed multi-objective optimization scheme is within a reasonable range which shows the low complexity nature of the scheme.

Fig. 9 further emphasizes the utility of our proposed method by plotting the total energy efficiency of the network against increasing values of available BS transmit power. From these

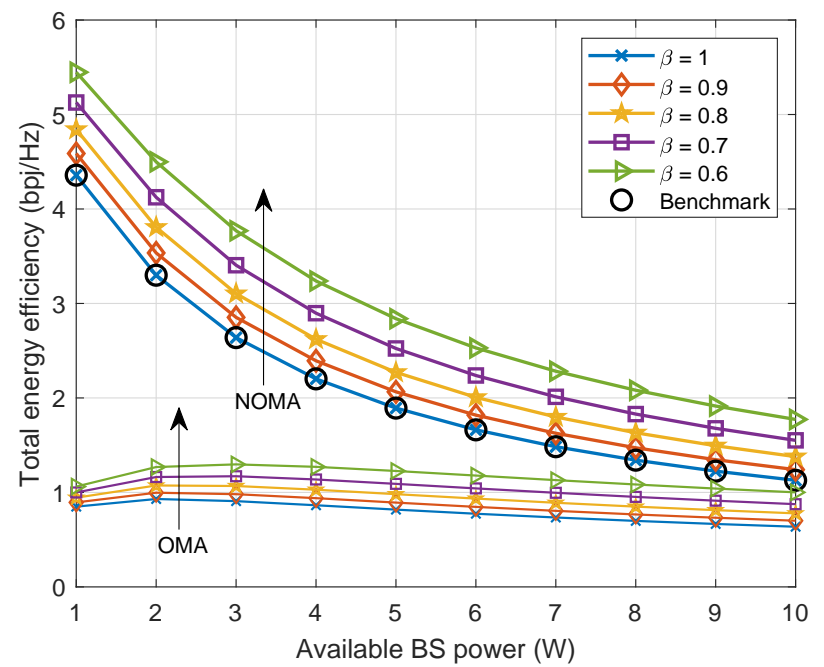

Figure 9. Total energy efficiency of the network against the available BS transmit power for $M=3, \mathrm{PC}=1$.

results, one can be observed that the total energy efficiency of the network decreases with an increase in the transmit power. However, the reduction in energy efficiency is far more for the case of OMA and benchmark NOMA optimization scheme. More specifically, for a fixed value of transmit power, i.e., 4 $\mathrm{W}$ and $\beta=0.6$, the energy efficiency of OMA is $1.3 \mathrm{bpj} / \mathrm{Hz}$, while for the same values, the energy efficiency of NOMA increases up to $3.3 \mathrm{bpj} / \mathrm{Hz}$. This shows the effectiveness of joint spectral and energy efficiency optimization.

Lastly, to see the impact of circuit power consumption (PC) on the total energy efficiency of the network, in Fig. 10, we provide the total energy efficiency of $M$ users for NOMA and OMA network with different values of PC consumption. One can observe that the system energy efficiency increases when the PC consumption decreases. More specifically, for a fixed value of transmit power, i.e., $3 \mathrm{~W}$ and $\mathrm{PC}=0.3$, the total energy efficiency of our joint power allocation scheme is 5 $\mathrm{bpj} / \mathrm{Hz}$, while for the same values, the total energy efficiency of OMA network is $1.7 \mathrm{bpj} / \mathrm{Hz}$. It is show the effectiveness of our proposed NOMA multi-objective optimization technique over its counterpart traditional OMA scheme.

\section{CONCLUSion}

Joint optimization of spectral and energy efficiency is critical for future NOMA systems. In this regard, this work has provided a novel technique for jointly improving the energy and spectral efficiency of multi-user NOMA systems. In particular, we have considered a downlink communication system and have shown that the proposed multi-objective optimization methodology works very well for NOMA systems. We have also compared the simulation results of our joint optimization scheme with the benchmark single-objective technique and conventional OMA technique. Our results have confirmed the superiority of the proposed multi-objective optimization technique over benchmark NOMA and OMA while maintaining a reasonable level of complexity. 


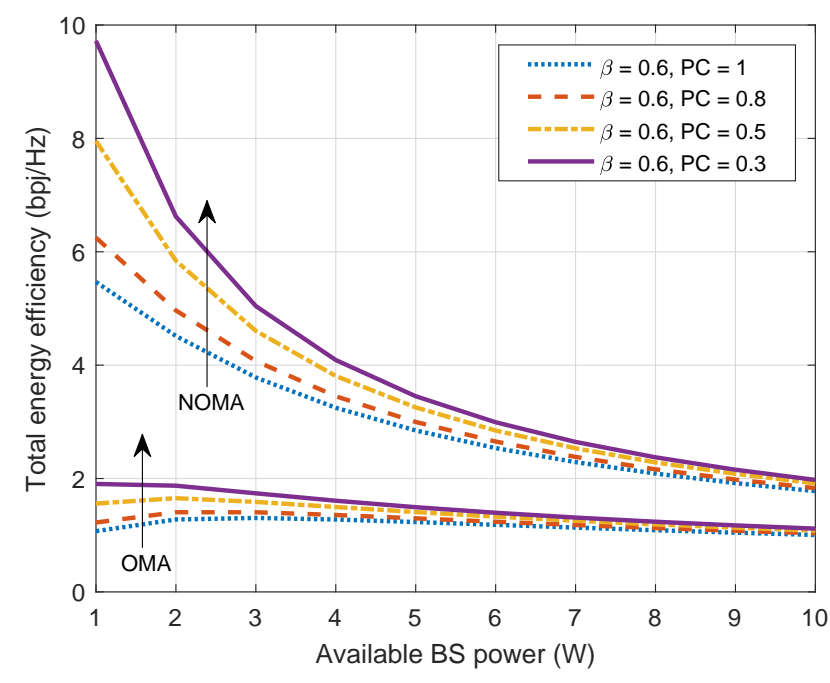

Figure 10. Total energy efficiency of the network with different PC consumptions versus the increasing values of available BS power for $M=3$, $\beta=0.6$.

Although the above-mentioned results are self-contained, in the future, we aim to extend them by incorporating channel estimation errors in downlink transmission. Afterward, we also aim to investigate the performance of our proposed multiobjective problem for cooperative downlink NOMA systems. These interesting and challenging problems will be addressed in future works.

\section{APPENDIX A}

\section{PROOF OF PROPOSITION 1}

Here, we provide the convexity proof of our proposed joint optimization problem. To do so, we first derive the Hessian matrix and then prove it as a negative definite. Note that the Hessian matrix is negative definite when its leading principle minors are alternate in sign, i.e., negative for odd number and positive for those of even number.

Let us derive the objective function of our proposed joint problem as

$$
\max _{p_{m}} \beta\left(\sum_{m=1}^{M} R_{m}\right)-(1-\beta)\left(\sum_{m=1}^{M} p_{m}\right)
$$

where it can be seen that the second segment - 1 $\beta)\left(\sum_{m=1}^{M} p_{m}\right)$ in (17) is a linear function of $p_{m}$. The first segment $\beta\left(\sum_{m=1}^{M} R_{m}\right)$ in (17) is strictly concave-convex.

Let $M=3$ downlink NOMA network where the channel powers are ordered as $\left|h_{1}\right|^{2} \leq\left|h_{2}\right|^{2} \leq\left|h_{3}\right|^{2}$, thereby we can write the sum rate as:

$$
\begin{aligned}
& R_{\text {sum }}=\log _{2}\left(1+\frac{p_{1}\left|h_{1}\right|^{2}}{\left(p_{2}+p_{3}\right)\left|h_{1}\right|^{2}+\sigma^{2}}\right) \\
& +\log _{2}\left(1+\frac{p_{2}\left|h_{2}\right|^{2}}{p_{3}\left|h_{2}\right|^{2}+\sigma^{2}}\right)+\log _{2}\left(1+\frac{p_{3}\left|h_{3}\right|^{2}}{\sigma^{2}}\right)
\end{aligned}
$$

Now following (18), the Hessian matrix $\coprod$ can be derived as

$$
\coprod=\left[\begin{array}{lll}
\left(\frac{\partial R_{\text {sum }}}{\partial p_{1}}\right) \frac{\partial}{\partial p_{1}} & \left(\frac{\partial R_{\text {sum }}}{\partial p_{1}}\right) \frac{\partial}{\partial p_{2}} & \left(\frac{\partial R_{\text {sum }}}{\partial p_{1}}\right) \frac{\partial}{\partial p_{3}} \\
\left(\frac{\partial R_{\text {sum }}}{\partial p_{2}}\right) \frac{\partial}{\partial p_{1}} & \left(\frac{\partial R_{\text {sum }}}{\partial p_{2}}\right) \frac{\partial}{\partial p_{2}} & \left(\frac{\partial R_{\text {sum }}}{\partial p_{2}}\right) \frac{\partial}{\partial p_{3}} \\
\left(\frac{\partial R_{\text {sum }}}{\partial p_{3}}\right) \frac{\partial}{\partial p_{1}} & \left(\frac{\partial R_{\text {sum }}}{\partial p_{3}}\right) \frac{\partial}{\partial p_{2}} & \left(\frac{\partial R_{\text {sum }}}{\partial p_{3}}\right) \frac{\partial}{\partial p_{3}}
\end{array}\right]
$$

After solving for derivations, we obtain

$$
\coprod=\left[\begin{array}{ccc}
-\Pi & -\Pi & -\Pi \\
-\Pi & -\Pi+\Pi^{\prime}-\Upsilon & -\Pi+\Pi^{\prime}-\Upsilon \\
-\Pi & -\Pi+\Pi^{\prime}-\Upsilon & -\Pi+\Pi^{\prime}-\Upsilon+\Upsilon^{\prime}-\Psi
\end{array}\right]
$$

where

$$
\begin{gathered}
\Pi=\left(\frac{\left|h_{1}\right|^{2}}{\left(p_{1}+p_{2}+p_{3}\right)\left|h_{1}\right|^{2}+\sigma^{2}}\right)^{2}, \\
\Pi^{\prime}=\left(\frac{\left|h_{1}\right|^{2}}{\left(p_{2}+p_{3}\right)\left|h_{1}\right|^{2}+\sigma^{2}}\right)^{2}, \\
\Upsilon=\left(\frac{\left|h_{2}\right|^{2}}{\left(p_{2}+p_{3}\right)\left|h_{2}\right|^{2}+\sigma^{2}}\right)^{2} \\
\Upsilon^{\prime}=\left(\frac{\left|h_{2}\right|^{2}}{p_{3}\left|h_{2}\right|^{2}+\sigma^{2}}\right)^{2} \\
\Psi=\left(\frac{\left|h_{3}\right|^{2}}{p_{3}\left|h_{2}\right|^{2}+\sigma^{2}}\right)^{2} .
\end{gathered}
$$

The first order principle in (20) can be expressed as

$$
\operatorname{det}\left|\coprod_{1}\right|=-\Pi
$$

As such, the second order principle minor is given by

$$
\begin{aligned}
\operatorname{det}\left|\coprod_{2}\right| & =\Pi\left(\Upsilon-\Pi^{\prime}\right) \\
& =\Pi\left(\left(\frac{\left|h_{2}\right|^{2}}{p_{2}\left|h_{2}\right|^{2}+\sigma^{2}}\right)^{2}-\left(\frac{\left|h_{1}\right|^{2}}{p_{2}\left|h_{1}\right|^{2}+\sigma^{2}}\right)^{2}\right)
\end{aligned}
$$

By taking LCM, we get

$$
=\Pi\left(\frac{\left(2 p_{2}\left|h_{1}\right|^{2}\left|h_{2}\right|^{2}+\left|h_{1}\right|^{2}+\left|h_{2}\right|^{2}\right)\left(\left|h_{2}\right|^{2}-\left|h_{1}\right|^{2}\right)}{\left(p_{2}\left|h_{1}\right|^{2}+\sigma^{2}\right)^{2}\left(p_{2}\left|h_{2}\right|^{2}+\sigma^{2}\right)^{2}}\right) .
$$

Following the same trend, we can derive the third principle minor as

$$
\begin{aligned}
\operatorname{det}\left|\coprod_{3}\right| & =-\Pi\left(\Upsilon-\Pi^{\prime}\right)\left(\Psi-\Upsilon^{\prime}\right) \\
& =-\Pi\left(\left(\frac{\left|h_{2}\right|^{2}}{p_{2}\left|h_{2}\right|^{2}+\sigma^{2}}\right)^{2}-\left(\frac{\left|h_{1}\right|^{2}}{p_{2}\left|h_{1}\right|^{2}+\sigma^{2}}\right)^{2}\right) \\
& \times\left(\left(\frac{\left|h_{3}\right|^{2}}{p_{3}\left|h_{2}\right|^{2}+\sigma^{2}}\right)^{2}-\left(\frac{\left|h_{2}\right|^{2}}{p_{3}\left|h_{2}\right|^{2}+\sigma^{2}}\right)^{2}\right),
\end{aligned}
$$


By taking LCM, we obtain

$$
\begin{aligned}
& =-\Pi \times \\
& \left(\frac{\left(2\left(p_{2}+p_{3}\right)\left|h_{1}\right|^{2}\left|h_{2}\right|^{2}+\left|h_{1}\right|^{2}+\left|h_{2}\right|^{2}\right)\left(\left|h_{2}\right|^{2}-\left|h_{1}\right|^{2}\right)}{\left(\left(p_{2}+p_{3}\right)\left|h_{1}\right|^{2}+\sigma^{2}\right)^{2}\left(\left(p_{2}+p_{3}\right)\left|h_{2}\right|^{2}+\sigma^{2}\right)^{2}}\right) \\
& \times\left(\frac{\left(2 p_{3}\left|h_{2}\right|^{2}\left|h_{3}\right|^{2}+\left|h_{2}\right|^{2}+\left|h_{3}\right|^{2}\right)\left(\left|h_{3}\right|^{2}-\left|h_{2}\right|^{2}\right)}{\left(p_{3}\left|h_{2}\right|^{2}+\sigma^{2}\right)^{2}\left(p_{3}\left|h_{3}\right|^{2}+\sigma^{2}\right)^{2}}\right) \cdot
\end{aligned}
$$

where $\Pi, \Pi^{\prime}, \Upsilon, \Upsilon^{\prime}$ and $\Psi$ are all positive. Therefore, the first and second order principle minors are negative and the third one is positive. Thus, our proposed joint optimization problem is concave-convex function.

\section{APPENDIX B}

\section{DERIVATION OF CLOSED FORM EXPRESSION}

To efficiently perform the SIC process, the channel gains of users are assumed as $\left|h_{1}\right|^{2} \leq\left|h_{2}\right|^{2} \leq \ldots \leq\left|h_{M}\right|^{2}$. When $m=1$, it represents the weakest user, i.e., $U_{1}$ and directly decodes the multiplex signal from BS without applying SIC technique. Note that in such case, the other signals are acted as an interference for $U_{1}$. Thus, the closed-form expression for $U_{1}$ without SIC can be derived as

$$
\begin{aligned}
\frac{\partial \mathcal{L}(.)}{\partial p_{1}} & =\left(\beta+\lambda_{1}\right)\left(\frac{\left|h_{1}\right|^{2}}{\sum_{n=1}^{M} p_{n}\left|h_{1}\right|^{2}+\sigma^{2}}\right)+\beta+\eta_{1}\left|h_{2}\right|^{2} \\
& -\mu_{1}-1=0
\end{aligned}
$$

Now we can obtain $p_{1}^{*}$ as

$$
p_{1}^{*}=\left[\frac{\left(\beta+\lambda_{1}\right)\left|h_{1}\right|^{2}-\phi_{1}\left(p_{n}\left|h_{1}\right|^{2}\right)-\sigma^{2} \phi_{1}}{\phi_{1}\left|h_{1}\right|^{2}}\right]^{+} .
$$

where $\phi_{1}=\mu_{1}+1-\beta-\eta_{1}\left|h_{2}\right|^{2}$.

For second user when $m=2, U_{2}$ first decodes and removes the signal of $U_{1}$ and then decodes its signal using SIC. However, $U_{2}$ can not remove the signal of any user when $m=2+1$. The solution of $U_{2}$ can be then derived as

$$
\begin{aligned}
\frac{\partial \mathcal{L}(.)}{\partial p_{2}} & =\left(\beta+\lambda_{2}\right)\left(\frac{\left|h_{2}\right|^{2}}{\sum_{n=2}^{M} p_{n}\left|h_{2}\right|^{2}+\sigma^{2}}\right)+\left(\beta+\lambda_{1}\right) \\
& \times\left(\frac{-\left|h_{1}\right|^{2}}{\sum_{n=2}^{M} p_{n}\left|h_{1}\right|^{2}+\sigma^{2}}\right)+\eta_{2}\left|h_{3}\right|^{2}+\beta-\eta_{1}\left|h_{2}\right|^{2} \\
& -\mu_{2}-1=0 .
\end{aligned}
$$

Then, the optimal value can be obtained as

$$
p_{2}^{*}=\left[\frac{\left(\beta+\lambda_{2}\right)\left|h_{2}\right|^{2}-\phi_{2}\left(p_{n}\left|h_{2}\right|^{2}-\sigma^{2} \phi_{2}\right)}{\phi_{2}\left|h_{2}\right|^{2}}\right]^{+}
$$

where

$$
\begin{aligned}
\phi_{2} & =\eta_{1}\left|h_{2}\right|^{2}+\mu_{2}+1-\eta_{2}\left|h_{3}\right|^{2}-\beta-\left(\beta+\lambda_{1}\right) \\
& \times\left(\frac{-\left|h_{1}\right|^{2}}{\sum_{n=2}^{M} p_{n}\left|h_{1}\right|^{2}+\sigma^{2}}\right) .
\end{aligned}
$$

Therefore, by deduction method, the optimal power of $U_{m}$ can be derived as (10).

\section{REFERENCES}

[1] M. Vaezi, G. Amarasuriya, Y. Liu, A. Arafa, F. Fang, and Z. Ding, "Interplay Between NOMA and Other Emerging Technologies: A Survey," IEEE Transactions on Cognitive Communications and Networking, pp. $1-1,2019$.

[2] N. Zhao, X. Pang, Z. Li, Y. Chen, F. Li, Z. Ding, and M.-S. Alouini, "Joint trajectory and precoding optimization for UAV-assisted NOMA networks," IEEE Transactions on Communications, 2019.

[3] W. U. Khan, Z. Yu, S. Yu, G. A. S. Sidhu, and J. Liu, "Efficient power allocation in downlink multi-cell multi-user NOMA networks," IET Communications, vol. 13, no. 4, pp. 396-402, 2019.

[4] F. Jameel, S. Wyne, S. J. Nawaz, Z. Chang, and T. Ristaniemi, "Outage Analysis of Relay-Aided Non-Orthogonal Multiple Access with Partial Relay Selection," in 2018 IEEE Globecom Workshops (GC Wkshps), Dec 2018, pp. 1-6.

[5] J. Shi, W. Yu, Q. Ni, W. Liang, Z. Li, and P. Xiao, "Energy efficient resource allocation in hybrid non-orthogonal multiple access systems," IEEE Transactions on Communications, 2019.

[6] H. Al-Obiedollah, K. Cumanan, J. Thiyagalingam, A. G. Burr, Z. Ding, and O. A. Dobre, "Energy efficient beamforming design for MISO nonorthogonal multiple access systems," IEEE Transactions on Communications, 2019.

[7] H. Zhang, F. Fang, J. Cheng, K. Long, W. Wang, and V. C. Leung, "Energy-efficient resource allocation in NOMA heterogeneous networks," IEEE Wireless Communications, vol. 25, no. 2, pp. 48-53, 2018.

[8] Q. Liu, T. Lv, and Z. Lin, "Energy-efficient transmission design in cooperative relaying systems using NOMA," IEEE Communications Letters, vol. 22, no. 3, pp. 594-597, March 2018.

[9] L. Pei, Z. Yang, C. Pan, W. Huang, M. Chen, M. Elkashlan, and A. Nallanathan, "Energy-efficient D2D communications underlaying NOMA-based networks with energy harvesting," IEEE Communications Letters, vol. 22, no. 5, pp. 914-917, May 2018.

[10] Q. Liu, H. Gao, F. Tan, T. Lv, and Y. Lu, "Energy efficient resource allocation in multi-user downlink non-orthogonal multiple access systems," in IEEE Global Communications Conference (GLOBECOM), Dec 2017, pp. 1-6.

[11] Y. Zhang, H. Wang, T. Zheng, and Q. Yang, "Energy-efficient transmission design in non-orthogonal multiple access," IEEE Transactions on Vehicular Technology, vol. 66, no. 3, pp. 2852-2857, March 2017.

[12] F. Fang, H. Zhang, J. Cheng, S. Roy, and V. C. M. Leung, "Joint user scheduling and power allocation optimization for energy-efficient NOMA systems with imperfect CSI," IEEE Journal on Selected Areas in Communications, vol. 35, no. 12, pp. 2874-2885, Dec 2017.

[13] H. Zhang, B. Wang, C. Jiang, K. Long, A. Nallanathan, V. C. M. Leung, and H. V. Poor, "Energy efficient dynamic resource optimization in NOMA system," IEEE Transactions on Wireless Communications, vol. 17, no. 9, pp. 5671-5683, Sept 2018.

[14] D. Ni, L. Hao, Q. T. Tran, and X. Qian, "Transmit power minimization for downlink multi-cell multi-carrier NOMA networks," IEEE Соттиnications Letters, pp. 1-1, 2018.

[15] F. Fang, J. Cheng, and Z. Ding, "Joint energy efficient subchannel and power optimization for a downlink NOMA heterogeneous network," IEEE Transactions on Vehicular Technology, pp. 1-1, 2018.

[16] B. Xu, Y. Chen, J. R. Carrión, and T. Zhang, "Resource allocation in energy-cooperation enabled two-tier NOMA HetNets toward green 5G," IEEE Journal on Selected Areas in Communications, vol. 35, no. 12, pp. 2758-2770, 2017.

[17] X. Song, L. Dong, J. Wang, L. Qin, and X. Han, "Energy Efficient Power Allocation for Downlink NOMA Heterogeneous Networks With Imperfect CSI," IEEE Access, vol. 7, pp. 39 329-39340, 2019.

[18] J. Cui, Y. Liu, Z. Ding, P. Fan, and A. Nallanathan, "QoE-based resource allocation for multi-cell NOMA networks," IEEE Transactions on Wireless Communications, vol. 17, no. 9, pp. 6160-6176, 2018.

[19] X. Sun, N. Yang, S. Yan, Z. Ding, D. W. K. Ng, C. Shen, and Z. Zhong, "Joint beamforming and power allocation in downlink NOMA multiuser MIMO networks," IEEE Transactions on Wireless Communications, vol. 17, no. 8, pp. 5367-5381, 2018.

[20] B. Chen, Y. Chen, Y. Chen, Y. Cao, N. Zhao, and Z. Ding, "A novel spectrum sharing scheme assisted by secondary NOMA relay," IEEE Wireless Communications Letters, vol. 7, no. 5, pp. 732-735, 2018.

[21] M. F. Hanif, Z. Ding, T. Ratnarajah, and G. K. Karagiannidis, "A minorization-maximization method for optimizing sum rate in the downlink of non-orthogonal multiple access systems," IEEE Transactions on Signal Processing, vol. 64, no. 1, pp. 76-88, 2016. 
[22] S. Zhang, B. Di, L. Song, and Y. Li, "Sub-channel and power allocation for non-orthogonal multiple access relay networks with amplify-andforward protocol," IEEE Transactions on Wireless Communications, vol. 16, no. 4, pp. 2249-2261, 2017.

[23] Y. Fu, L. Salaün, C. W. Sung, C. S. Chen, and M. Coupechoux, "Double iterative waterfilling for sum rate maximization in multicarrier NOMA systems," in 2017 IEEE International Conference on Communications (ICC). IEEE, 2017, pp. 1-6.

[24] C. Xue, Q. Zhang, Q. Li, and J. Qin, "Joint power allocation and relay beamforming in nonorthogonal multiple access amplify-and-forward relay networks," IEEE Transactions on Vehicular Technology, vol. 66 , no. 8, pp. 7558-7562, 2017.

[25] B. Di, L. Song, and Y. Li, "Sub-channel assignment, power allocation, and user scheduling for non-orthogonal multiple access networks," IEEE Transactions on Wireless Communications, vol. 15, no. 11, pp. 76867698, 2016.

[26] G. Liang, Q. Zhu, J. Xin, Y. Feng, and T. Zhang, "Joint user-channel assignment and power allocation for non-orthogonal multiple access relaying networks," IEEE Access, vol. 7, pp. 30 361-30372, 2019.

[27] Z. Song, Q. Ni, and X. Sun, "Distributed power allocation for nonorthogonal multiple access heterogeneous networks," IEEE Communications Letters, vol. 22, no. 3, pp. 622-625, 2018.

[28] J. Zhao, Y. Liu, K. K. Chai, A. Nallanathan, Y. Chen, and Z. Han, "Spectrum allocation and power control for non-orthogonal multiple access in HetNets," IEEE Transactions on Wireless Communications, vol. 16, no. 9, pp. 5825-5837, 2017.

[29] D. Ni, L. Hao, Q. T. Tran, and X. Qian, "Power allocation for downlink NOMA heterogeneous networks," IEEE Access, vol. 6, pp. 26742 26752, 2018.

[30] W. Xu, R. Qiu, and X.-Q. Jiang, "Resource Allocation in Heterogeneous Cognitive Radio Network With Non-Orthogonal Multiple Access," IEEE Access, vol. 7, pp. 57488-57 499, 2019.

[31] E. Bedeer, O. A. Dobre, M. H. Ahmed, and K. E. Baddour, "A multiobjective optimization approach for optimal link adaptation of OFDM-based cognitive radio systems with imperfect spectrum sensing," IEEE Transactions on Wireless Communications, vol. 13, no. 4, pp. 2339-2351, 2014.

[32] N. Gunantara, "A review of multi-objective optimization: Methods and its applications," Cogent Engineering, vol. 5, no. 1, p. 1502242, 2018.

[33] M. Naeem, A. S. Khwaja, A. Anpalagan, and M. Jaseemuddin, "Green cooperative cognitive radio: A multiobjective optimization paradigm," IEEE Systems Journal, vol. 10, no. 1, pp. 240-250, 2014.

[34] K. Miettinen, Nonlinear multiobjective optimization. Springer Science \& Business Media, 2012, vol. 12.

[35] W. U. Khan, F. Jameel, T. Ristaniemi, B. M. Elhalawany, and J. Liu, "Efficient Power Allocation for Multi-Cell Uplink NOMA Network," in 2019 IEEE 89th Vehicular Technology Conference (VTC2019-Spring). IEEE, 2019, pp. 1-5.

[36] Z. Ali, G. A. S. Sidhu, M. Waqas, and F. Gao, "On fair power optimization in nonorthogonal multiple access multiuser networks," Transactions on Emerging Telecommunications Technologies, vol. 29, no. 12, p. e3540, 2018.

[37] R. T. Marler and J. S. Arora, "The weighted sum method for multiobjective optimization: new insights," Structural and multidisciplinary optimization, vol. 41, no. 6, pp. 853-862, 2010.

[38] M. R. Mili, K. A. Hamdi, F. Marvasti, and M. Bennis, "Joint optimization for optimal power allocation in OFDMA femtocell networks," IEEE Communications Letters, vol. 20, no. 1, pp. 133-136, 2016.

[39] R. T. Marler and J. S. Arora, "Survey of multi-objective optimization methods for engineering," Structural and multidisciplinary optimization, vol. 26, no. 6, pp. 369-395, 2004.

[40] A. Ali, A. Baig, G. M. Awan, W. U. Khan, Z. Ali, and G. A. S. Sidhu, "Efficient Resource Management for Sum Capacity Maximization in 5G NOMA Systems," Applied System Innovation, vol. 2, no. 3, p. 27, 2019

[41] W. U. Khan, "Maximizing physical layer security in relay-assisted multicarrier nonorthogonal multiple access transmission," Internet Technology Letters, vol. 2, no. 2, p. e76, 2019.

[42] M. S. Ali, E. Hossain, A. Al-Dweik, and D. I. Kim, "Downlink power allocation for CoMP-NOMA in multi-cell networks," IEEE Transactions on Communications, vol. 66, no. 9, pp. 3982-3998, 2018.

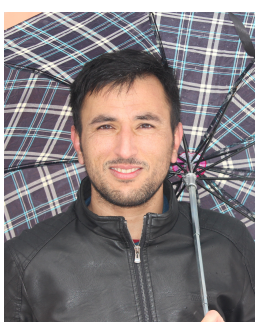

Wali Ullah Khan received his BS (Honours) in Telecommunication from the University of Science and Technology Bannu, Khyber Pakhtunkhwa, Pakistan, in 2014, and the Master's degree in Electrical Engineering from the Islamabad Campus of COMSATS Institute of Information Technology (currently known COMSATS University), Pakistan, in 2017. Currently, he is with the School of Information Science and Engineering, Shandong University, Qingdao, China, where his research interests include convex/ non-convex optimization, non-orthogonal multiple access, energy/ spectral efficiency, physical layer security, and backscatter communications. He is an active reviewer for several SCI journals such as IEEE Wireless Communications, EURASIP Journal on Wireless Communications and Networking, IEEE Access, and Physical Communication.

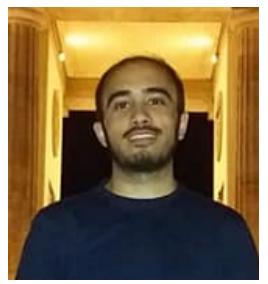

Furqan Jameel received his BS in Electrical Engineering (under ICT R\&D funded Program) in 2013 from the Lahore Campus of COMSATS Institute of Information Technology (CIIT), Pakistan. In 2017, he received his Master's degree in Electrical Engineering (funded by prestigious Higher Education Commission Scholarship) at the Islamabad Campus of CIIT. In September 2018, he visited Simula Research Laboratory and the University of Oslo, Norway. From 2018 to 2019, he was with the University of Jyväskylä, Finland, and Nokia Bell Labs, Espoo, where he worked as a researcher and a summer trainee, respectively. Currently, he is with the Department of Communications and Networking, Aalto University, 02150 Espoo, Finland, where his research interests include modeling and performance enhancement of vehicular networks, machine/ deep learning, ambient backscatter communications, and wireless power transfer. $\mathrm{He}$ is the recipient of outstanding reviewer award 2017 from Elsevier.

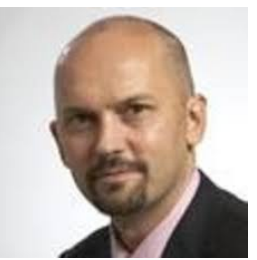

Tapani Ristaniemi received his M.Sc. in 1995 (Mathematics), Ph.Lic. in 1997 (Applied Mathematics) and Ph.D. in 2000 (Wireless Communications), all from the University of Jyväskylä, Jyväskylä, Finland. In 2001 he was appointed as Professor in the Department of Mathematical Information Technology, University of Jyväskylä. In 2004 he moved to the Department of Communications Engineering, Tampere University of Technology, Tampere, Finland, where he was appointed as Professor in Wireless Communications. In 2006 he moved back to University of Jyväskylä to take up his appointment as Professor in Computer Science. He is an Adjunct Professor of Tampere University of Technology. In 2013 he was a Visiting Professor in the School of Electrical and Electronic Engineering, Nanyang Technological University, Singapore. $\mathrm{He}$ has authored or co-authored over 150 publications in journals, conference proceedings and invited sessions. He served as a Guest Editor of IEEE Wireless Communications in 2011 and currently he is an Editorial Board Member of Wireless Networks and International Journal of Communication Systems. His research interests are in the areas of brain and communication signal processing and wireless communication systems research.

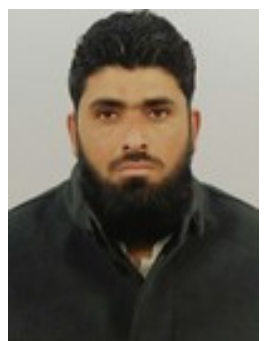

Shafiullah Khan received the M.Sc. degree in Electronics from the University of Peshawar, Pakistan, in 2008, and Ph.D. degrees in Electronic Engineering from Zhejiang University, Hangzhou, China. Currently, he is with the Department of Electronics, Islamia College University Peshawar, Pakistan, where is an Assistant Professor. He has published more than 20 research papers in the national and international Journals. He is an active reviewer of several International journals. His research interests include Numerical methods for Electromagnetic, Evolutionary algorithms, and Artificial intelligence. 


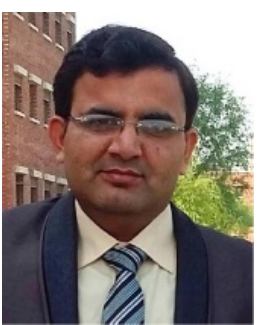

Guftaar Ahmad Sardar Sidhu received the B.Sc. degree in computer engineering from the COMSATS Institute of Information Technology, Islamabad, Pakistan, in 2006 and the M.Sc. and Ph.D. degrees in electrical engineering from Jacobs University, Bremen, Germany, in 2009 and 2012, respectively.

$\mathrm{He}$ is currently an Assistant Professor with the Department of Electrical and Computer Engineering, COMSATS University Islamabad. His research interests include cooperative wireless communication, cognitive radio networks, multiuser multicarrier systems, heterogeneous networks, physical-layer security, energy harvesting, non-orthogonal multiple access schemes, energy management in smart grids, and economic dispatch in future renewable integrated power systems. Dr. Sidhu received the Best Paper Award at the IEEE International Wireless Communication and Signal Processing Conference in 2010. He has published more than 50 research papers in international journals and peer-reviewed conferences.

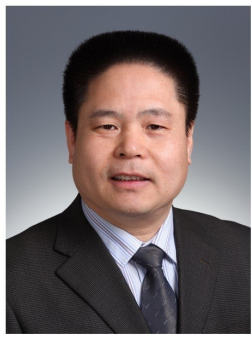

Ju Liu (M'02-SM'09) received B.S. degree and M.S. degree both in Electronic Engineering from Shandong University (SDU), Jinan, China, in 1986 and 1989, respectively; and the Ph.D. degree in Signal Processing from Southeast University (SEU), Nanjing, China, in 2000. Since July 1989, he has been with the Department of Electronic Engineering of SDU.

From July, 2002 to December, 2003, he was a visiting professor in the Department of Signal Theory and Communication, Polytechnic University of Catalonia (UPC) and Telecommunications Technological Centre of Catalonia (CTTC), Barcelona, Spain. From November, 2005 to January, 2006, funded by DAAD, he was a Visiting Researcher in the Department of Communication Engineering, University of Bremen and the Department of Communication Systems, University of Duisburg-Essen in Germany. In March, 2018, funded by JSPS he was a Visiting Researcher in the College of Information Science and Engineering, Ritsumeikan University, Japan. From June to December, 2009, in July, 2012, and in January, 2019, he was a Senior Research Fellow in the Department of Electrical Engineering, University of Washington, Seattle, the Department of Electrical and Computer Engineering, University of California, San Diego, USA, and the school of Electronics and Computer Science, University of Southampton, UK, respectively.

Dr. Liu is the author or co-author of more than 200 journal and conference papers. He is the holder of the Program for New Century Excellent Talents in University (NCET) of China, and the holder of 3 conference Best Paper Awards, 5 national and local academic awards in communication and signal processing. He is an associate editor of the International Journal of Digital Crime and Forensics, a member of editorial committee of Journal of Swarm Intelligence Research and Journal of Electronics \& Information Technology in China. He has been an active reviewer for IEEE Trans. on Signal Processing, IEEE Signal Processing Letters, IEEE Trans. on Wireless Communications, IEEE Communications Letters, etc. His current research interests include space-time processing in wireless communication, blind signal separation and multimedia communications. 